\title{
Not another diabetic foot infection: A case report of Charcot's joint
}

\author{
Rina Jain MD, Mitchell Brown BSc MD MEd FRCSC, Erin L Boynton MD \\ Divisions of Orthopaedic and Plastic Surgery, Women's College Hospital, and Mount \\ Sinai Hospital, University of Toronto, Toronto, Ontario
}

R Jain, M Brown, EL Boynton. Not another diabetic foot infection: A case report of Charcot's joint. Can J Plast Surg 1996;4(4):205-208. Diabetic patients are susceptible to foot infections, including cellulitis and osteomyelitis. However, not every diabetic's foot with erythema, swelling and pain is the result of an infection. The presentation of a Charcot's joint (neuroarthropathy) may mimic that of an acute infection. In the absence of systemic signs of sepsis, a diagnosis of a Charcot's joint should be considered.

Key Words: Charcot's joint, Diabetic foot, Osteomyelitis

\section{Rapport de cas sur la neuroarthropathie de Charcot, et non pas sur une autre infection du pied diabétique}

RÉSUMÉ : Les patients diabétiques sont sensibles aux infection du pied, y compris à la cellulite et à l'ostéomyélite. Or un pied diabétique qui présente de l'érythème, de l'œdème et de la douleur n'est pas nécessairement infecté. Le tableau de la neuroarthropathie de Charcot peut avoir des similitudes avec celui d'une infection aiguë. En l'absence de signes de septicémie, on peut envisager un diagnostic de neuroarthropathie de Charcot.

Diabetic patients may be faced with complications of the foot and ankle secondary to a primary vascular impairment, peripheral sensory neuropathy and posttraumatic insult (1). The immunocompromised state seen in the diabetic patient can prolong the course of infections. Infections can then lead to osteomyelitis and the formation of abscesses. Orthopedic and plastic surgeons are frequently asked to see diabetic patients for the management of cellulitis and osteomyelitis. The diagnosis of osteomyelitis in a diabetic patient may be difficult because other conditions such as a neuropathic joint can mimic the severity of joint destruction. Diabetes is the most common condition associated with neuropathic (Charcot's) joints, occurring frequently in the foot and ankle (1). The following case illustrates a rapid progression of Charcot's joint that was originally thought to be osteomyelitis.

\section{CASE PRESENTATION}

A 62-year-old woman was admitted to hospital by the internal medicine service febrile and complaining of pain, erythema and swelling over the lateral aspect of her right foot. Her past medical history revealed diabetes mellitus which began in 1977. She had 
been insulin-dependent since 1985. Cellulitis was diagnosed, and the patient was placed on intravenous cloxacillin. The pain, swelling and fever resolved quickly, and she was subsequently switched to oral clindamycin and ciprofloxacin. She then developed generalized edema secondary to acute diabetic nephropathy and subsequent renal failure. Radiographs of her foot revealed tarsal joint destruction (Figure 1). A technetium ${ }^{99}$ pyrophosphate bone scan was positive in the area of the lateral ankle; however, a gallium scan was negative. Because of persistent erythema and swelling, she underwent incision and drainage on the lateral aspect of her foot for presumed abscess six weeks after her admission, but no pus was expressed. Results of intraoperative cultures were negative. The patient was discharged three days later, after recovery from renal failure and after showing signs of clinical improvement in her right foot. Nine days after discharge, the patient was readmitted to hospital with a new onset of mild pain, swelling and erythema over the lateral aspect of her foot (Figure 2). She had remained afebrile, and blood glucose levels had remained satisfactory since her discharge. She was taken to the operating room for incision and drainage of her foot. No pus was found, and there was evidence of necrotic tissue. A large collection of fluid consistent with a liquefied hematoma was noted.

Severe destruction of the tarsal bones and joints was noted intraoperatively such that there was a complete flail of the midfoot and hindfoot. The operative site was irrigated thoroughly and packed with povidone-iodine-soaked gauze. The radiographs of her foot on her second admission (Figure 3) indicated destruction of the tarsal and subtalar joints with new bone formation, consistent with a diagnosis of Charcot's joint. Comparison of the second admission radiographs with those completed on first admission (Figure 1) showed a striking progression of joint destruction over a period of nearly two months. The patient was placed in a below-knee cast and started on protected weightbearing, and all antibiotics were discontinued. Marked clinical improvement occurred over the next two months with decreasing erythema, swelling and pain. 

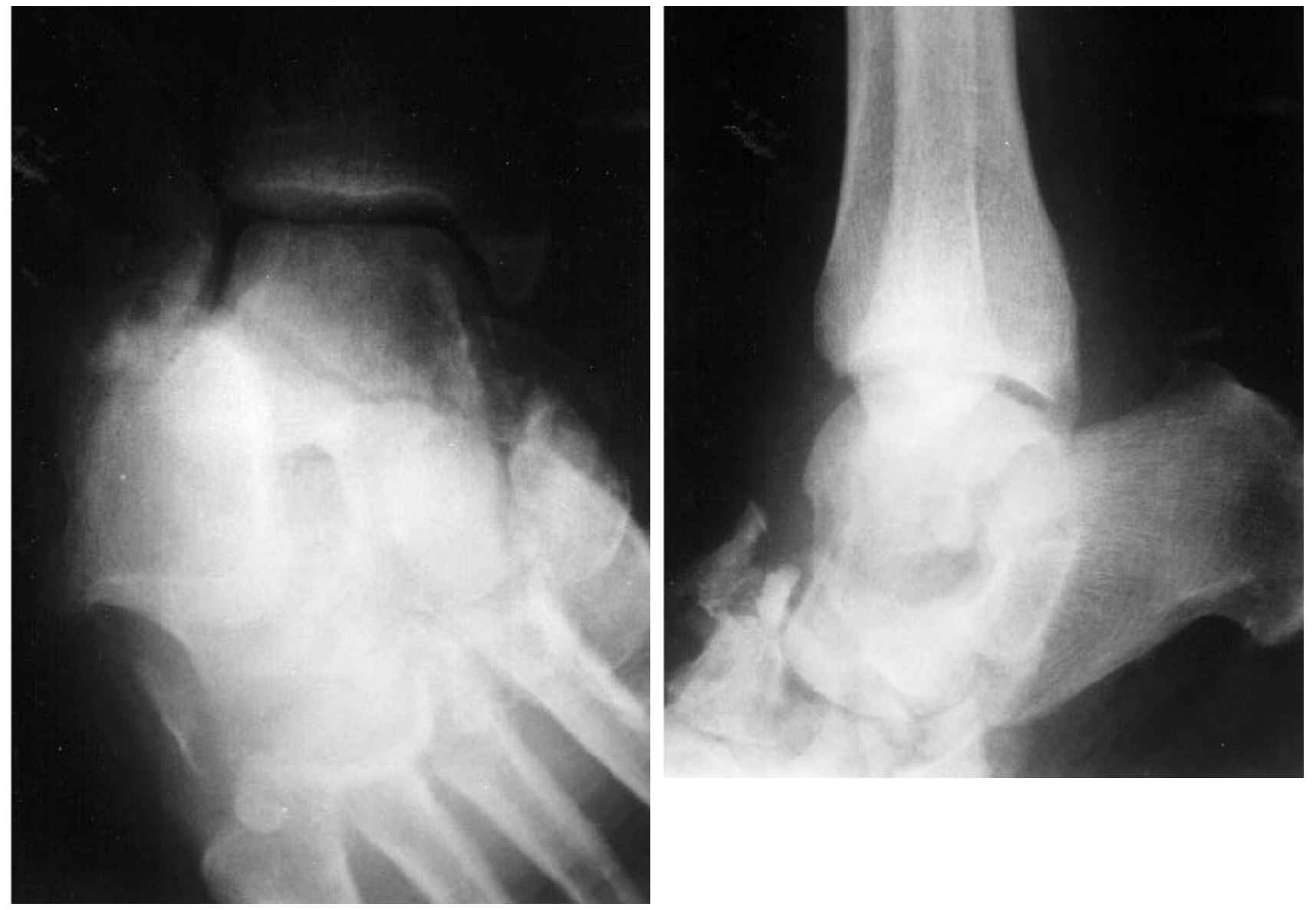

Figure 1) Radiographs of the patient's right foot at the time of initial presentation, demonsrtating destructive changes at he tarsaljoints with sclerosis and fragmentation.

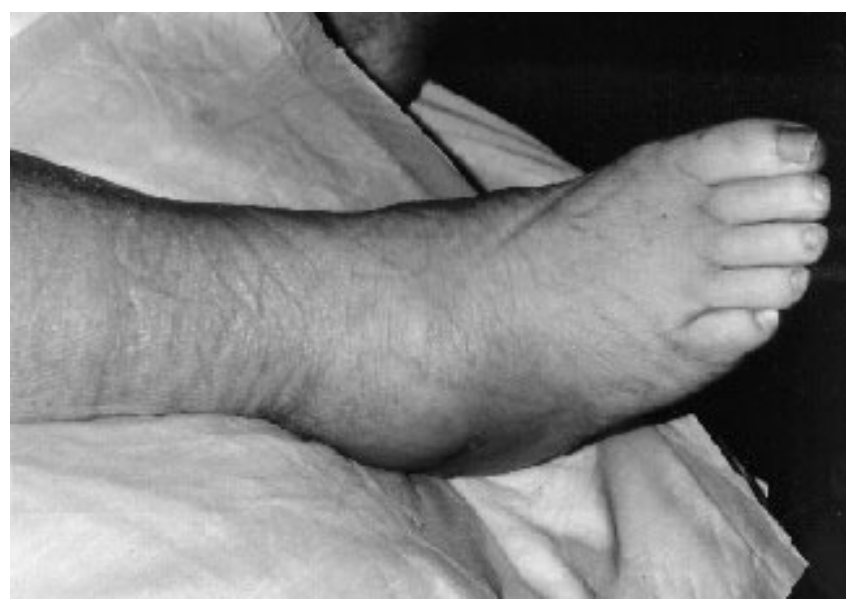

Figure 2) Photograph of the lateral aspect of the patient's right foot in the area of the mid-and hindfoot demonstrating erythema and swelling 

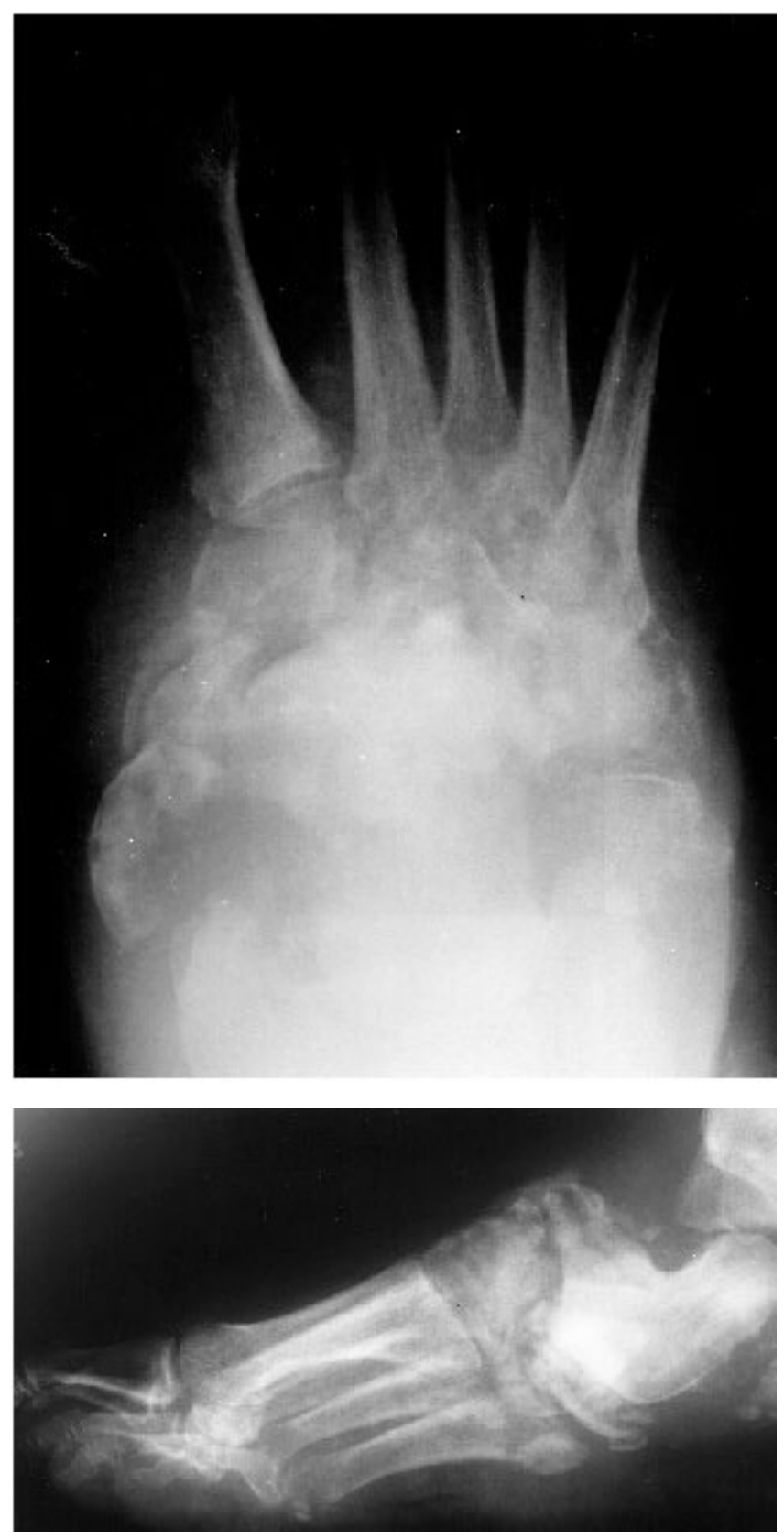

Figure 3) Radiographs of the patient's right foot two months after initial admission to hospital. Further destructive changes have occurred along her tarsal and metatarsals

\section{DISCUSSION}

Jean Martin Charcot originally described the clinical features of the neuropathic joint in 1868 in a patient with syphilis (2,3). In 1936, Jordan first reported the occurrence of Charcot's joint in association with diabetic neuropathy (4). The most common cause of Charcot's joints is diabetes mellitus, occurring in $0.1 \%$ to $6.8 \%$ of diabetics (4). Usually, 
patients with neuroarthropathy have had diabetes for 15 years, and are typically in their $50 \mathrm{~s}$ or $60 \mathrm{~s}(1,2,4)$. However, other conditions such as tabes dorsalis, leprosy, syringomyelia and spinal cord trauma can be associated with the development of neuropathic joints $(1,4,5)$.

Most diabetic neuropathic joints occur in the feet (5). In 60\% of Charcot's joints in diabetic patients, the tarsal-metatarsal joints are affected, with $30 \%$ occurring at the metatarsophalangeal joints and the remainder at the ankle (5). The pathophysiology of neuropathic joints is believed to be related to sensory denervation of the joints, secondary to the disease process, affecting the nerves to the joints. In diabetic patients, sensory neuropathy can occur, leading to altered joint pain sensation and proprioception $(2,4)$. The joint can be subject to repeated trauma (4), and unrecognized fractures occur and go unprotected (1). These fractures may then lead to the development of deformities (1). Radiographically, destructive changes followed by attempted fracture repair occur $(2,4)$. Joint debris, new bone formation in periarticular tissues and periosteum, and hemosiderin deposits secondary to frequent hemorrhages into the joint may be seen (2).

The acute presentation of a Charcot's joint may be confused with other conditions with similar features such as gout, cellulitis and osteomyelitis (3). In the present case, the patient showed signs of acute inflammation of the foot, including erythema, swelling and pain.

Diabetics are at a greater risk of developing osteomyelitis of the foot because of the presence of neuropathic ulcers which can advance into bone (1). The radiographs of a patient with osteomyelitis may be quite similar to those of a patient with Charcot's joint because bone and joint destruction may occur (1). New bone formation is found in both neuropathic joints and osteomyelitis, leading to positive bone scans in both situations (1). However, the extensive destruction seen in this patient's foot was not mirrored by the severity of her symptoms. The patient did have some pain and tenderness along her foot, but the severity did not match her radiographs. This is quite typical of neuropathic joints (4). Charcot's joints are commonly believed to be painless; however, pain may occasionally be a feature (3).

Three stages of neuroarthropathy have been defined. In the first stage, acute inflammation occurs, and radiographs reveal fragmentation of bone, leading to the confusion of this stage with acute osteomyelitis (3). The second stage marks the repair process with new bone formation and reduction of inflammatory symptoms and signs (3). In the final stage, bone and joint remodelling occur with consolidation of the fragmented bones $(3,4)$. On radiographs, the osseous fragments of neuropathic joints are supposedly well defined (6), whereas the fragments produced by osteomyelitis are osteoporotic with poorly defined margins (6).

This patient had rapid progression of destructive changes over a period of two months. She twice presented with acute inflammation. The amount of swelling was such that blistering and superficial ulceration occurred, as seen in acute neuroarthropathy (7). It is interesting to note that the patient initially improved after one month of antibiotic treatment and resting. However, after developing renal failure, her Charcot's joint changes advanced at a rapid rate. Diabetic patients with concomitant renal disease are particularly at risk for aggressive neuroarthropathic disease (3). As well, the patient's weight increased by $40 \mathrm{~kg}$ during this time. This may have contributed to increased loads on the already damaged joints. 
The differentiation between acute presentations of Charcot's joint and osteomyelitis is often difficult because the two conditions have many features in common. However, the lack of systemic sepsis or fever, significant hyperglycemia and leukocytosis may direct the diagnosis towards neuropathic joint. Radiographically, osteomyelitis appears to be similar to neuropathic joint with bone destruction and new bone formation in the later stages $(1,3,7,8)$. The use of bone scans may not differentiate the two conditions because any new bone formation will lead to increased uptake of the technetium-labelled pyrophosphate (8). As well, magnetic resonance imaging may not be able to distinguish Charcot's joints from osteomyelitis (7).

In summary, diabetic patients are at risk of developing foot complications secondary to peripheral neuropathy. Infections such as cellulitis, abscess formation and osteomyelitis continue to be the most common manifestations. However, in patients with atypical presentations where systemic signs of infection are absent, a diagnosis of Charcot's joint should be considered.

\section{REFERENCES}

1. Milgram JW. Osteomyelitis in the foot and ankle associated with diabetes mellitus. Clin Orthop 1993;296:50-7.

2. Bruckner FE, Howell A. Neuropathic joints. Semin Arthritis Rheum 1972;2:47-69. 3. Schon LC, Marks RM. The management of neuroarthropathic fracture-dislocations in the diabetic patient. Orthop Clin North Am 1995;26:375-92.

4. Esses S, Langer F, Gross A. Charcot's joints: A case report in a young patient with diabetes. Clin Orthop 1981;156:183-6.

5. Gupta R. A short history of neuropathic arthropathy. Clin Orthop 1993;296:43-9. 6. Slowman-Kovacs SD, Braunstein EM, Brandt KD. Rapidly progressive Charcot arthropathy following minor joint trauma in patients with diabetic neuropathy. Arthritis Rheum 1990;33:412-7.

7. Harrelson JM. The diabetic foot: Charcot arthropathy. In: Heckman JD, ed. Instructional Course Lectures, vol 42. Chicago: American Academy of Orthopedic Surgeons, 1993:141-6.

8. Harrelson JM. Management of the diabetic foot. Orthop Clin North Am 1989;20:60519. 